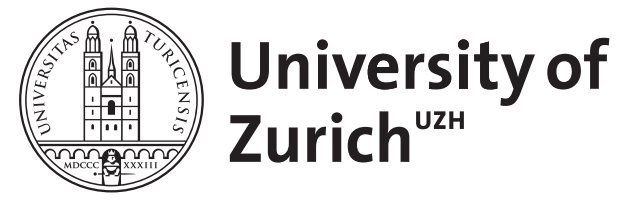

Zurich Open Repository and Archive

University of Zurich

University Library

Strickhofstrasse 39

CH-8057 Zurich

www.zora.uzh.ch

Year: 2018

Ein Mensch, den man nicht vergißt (1939)

Traupmann, Thomas

Posted at the Zurich Open Repository and Archive, University of Zurich

ZORA URL: https://doi.org/10.5167/uzh-162161

Book Section

Originally published at:

Traupmann, Thomas (2018). Ein Mensch, den man nicht vergißt (1939). In: Larcati, Arturo; Renoldner, Klemens; Wörgötter, Martina. Stefan-Zweig-Handbuch. Berlin / Boston: De Gruyter, 289-292. 
Neymeyr, Barbara (2004b): Konstruktion des Phantastischen. Die Krise der Identität in Kafkas Beschreibung eines Kampfes. Heidelberg: Winter.

Neymeyr, Barbara (2012): Aporien der Hasard-Leidenschaft im kulturanthropologischen Kontext. Die Inszenierungen des Glücksspiels in Stefan Zweigs Vierundzwanzig Stunden aus dem Leben einer Frau und in Arthur Schnitzlers Spiel im Morgengrauen. In: Gerrekens, Louis/Küpper, Achim (Hg.): Hasard. Der Spieler in der deutschsprachigen Literaturgeschichte. Würzburg: Königshausen \& Neumann, S. 141-168.

Neymeyr, Barbara: Voyeurismus als Erzählstrategie: Stefan Zweigs Novelle Unvermutete Bekanntschaft mit einem Handwerk und ihre intertextuellen Bezüge zu Grillparzers Erzählung Der arme Spielmann [in Vorbereitung].

Turner, David (1988): Moral Values and the Human Zoo. The „Novellen“ of Stefan Zweig. Hull: Hull Univ. Press.

Zilberfarb, Sacha (2013): Découverte inattendue d'un métier. Présentation. In: Zweig, Stefan: La confusion des sentiments et autres récits. Hg. v. Pierre Deshusses. Paris: Laffont, S. 10811082.

\subsection{Ein Mensch, den man nicht vergißt (1939)}

Thomas Traupmann

1. Entstehung . . . . . . . . . . . . . . . . . 289

2. Inhalt. . . . . . . . . . . . . . . . . . 290

3. Rezeption und Forschung . . . . . . . . . . . . . . . . 291

\section{Entstehung}

Der Erstdruck der kurzen Erzählung erfolgte unter dem Titel Anton, Friend of All the World. The Most Unforgettable Character I Ever Met im Oktober 1939 in dem englischsprachigen Reader's Digest. In deutscher Sprache wurde der Text erst postum im Jahr 1948 im Rahmen der ersten Reader's Digest-Ausgabe im deutschen Sprachraum - und damit an prominenter Stelle - als Ein Mensch, den man nicht vergisst. Ein Erlebnis veröffentlicht. Die Daniel A. Reed Library der Fredonia State University (Stefan Zweig Collection) verwahrt zwei Typoskripte, beide mit eigenhändigen Korrekturen des Autors: ein (textgenetisch früheres) deutschsprachiges unter dem Titel Die Kunst obne Sorgen zu leben und ein (späteres) englischsprachiges unter dem Titel The Man with the Secret of Happiness bzw. "The Master of the Art of Life (Living?)“, wie ein zusätzlicher Bleistiftvermerk besagt. Von den Typoskript-Fassungen weichen die weitaus kürzeren publizierten Fassungen deutlich ab. Die deutschsprachige Erstveröffentlichung gestaltet sich außerdem als Übersetzung des Erstdrucks von fremder Hand, für die Zweigs Typoskript-Fassung keine Rolle (mehr) spielt, wie etwa die Zecke („tick“) des Hundes, die auf dem Weg der Übersetzung verloren geht, belegt. Jene in Zweigs Nachlass aufgetauchte und von Knut Beck 1981 veröffentlichte Fassung unter dem Titel Anton, die der deutschsprachigen Erstveröffentlichung gekürzt um den ersten Absatz entspricht, ist folglich auch nicht auf das Jahr 1939 zu datieren (vgl. Zweig 1981, S. 393), sondern zeitlich später anzusetzen, auch wenn deren ursprünglicher Erscheinungskontext nach wie vor unbestimmbar bleibt. Obgleich keine weiteren Textzeugnisse eruiert werden konnten, ist außerdem davon auszugehen, dass auch 
die Kürzung der englischsprachigen Fassung für Reader's Digest von fremder Hand erfolgt ist - ,Verstümmelungen' betreffen schließlich auch die Novellen, die Zweig in den USA schreibt (vgl. Prater 1989, S. 1088). Da der vorliegende Artikel keinen Fassungsvergleich anstreben kann, bezieht er sich im Wesentlichen auf den Text der deutschsprachigen Erstveröffentlichung, der in weiterer Folge tradiert und der Rezeption zugänglich wurde.

Die Erzählung ist Zweigs erster Beitrag für Reader's Digest, 1940 folgt A Lesson from Rodin, 1941 erscheinen gemeinsam What Money Means to Me und Never Hesitate! Donald A. Prater betont die Relevanz des Honorars bei der Entstehung dieser Texte, verweist aber gleichzeitig auf Zweigs aufrichtiges Interesse an der Zeitschrift, für die er noch in den letzten Tagen in Petrópolis einen Beitrag erwogen habe (vgl. Prater 1989, S. 1089f.).

\section{Inhalt}

Ein namenloser Ich-Erzähler berichtet von der Begegnung mit dem „einzigartigen Menschen“ (Zweig GWE, Ein Mensch, den man nicht vergißt, S. 313) Anton, der seinen Hund von einem unversehens auftretenden Leiden befreit und anschließend ohne Dank oder Lohn einzufordern wieder verschwindet. Geld ist Anton, wie sich herausstellt, „ganz gleichgültig“ (S. 314), und er umgeht das „festgefügte[ ] Gesetz“ (S. 315), das die Ökonomie der nicht näher bezeichneten Kleinstadt bestimmt. Er macht überall auf notwendige Verbesserungen und Reparaturarbeiten aufmerksam, die ihm in der Regel auch sogleich überantwortet werden, und steht allen Leuten „,in Notfällen“ (S. 315) zu Diensten. Aus seinem Verhalten resultiert dabei eine Para-Ökonomie: Etabliert wird ein „ganz neues Wirtschaftssystem“, das auf der „Anständigkeit seiner Mitmenschen“ fußt und Anton ein „Guthaben moralischer Verpflichtungen“ verschafft (S. 316). Der Preis seiner Hilfsbereitschaft ist dann freilich - im Sinne von Gabe und Gegen-Gabe - ein „Gefühl der Verpflichtung“ (S. 316), ihre Kehrseite ein beachtliches Maß an „Macht“ (S. 316).

Gleichzeitig konstituiert Antons ökonomisches System eine „Gemeinschaft“ (S. 316), aus der sich der Erzähler zunächst ausgeschlossen fühlt, so wie man Anton auch „nicht einfach holen“ (S. 317) kann, sondern über ein Botenprinzip informieren muss. Nach Erledigung einiger Arbeiten im Haus des Erzählers wirbt Anton diesem mehrere Kleidungsstücke ab und bedenkt ihn dafür mit einem „schlichte[n]“ (S. 318), aber für ihn bedeutenden Kompliment. Ausgehend von der großen „moralische[n] Hilfe" (S. 318), die der Erzähler rückblickend von Anton empfangen haben will, wird gleichsam resümierend die Utopie einer Welt des gegenseitigen Vertrauens entworfen, in der es „keine Polizei, keine Gerichte, keine Gefängnisse und ... kein Geld“ (S. 319) gibt.

Sieht man von der fehlenden Prominenz seines Protagonisten ab, lässt sich der Text durchaus in die Nähe der Begegnungen mit Menschen, Büchern, Städten (1937) stellen; thematische Anklänge finden sich in Besuch bei den Milliarden (1932), wo Nationalbibliothek und Louvre „die wahren und unvergänglichen Schatzkammern unserer Welt" (Zweig GWE, Besuch bei den Milliarden, S. 337) genannt werden, sowie im erwähnten Beitrag What Money Means to Me, der resümiert: „[O]ur real security lies not in what we own, but in what we are and what we create out of ourselves“ (Zweig 1941a, S. 41). 


\section{Rezeption und Forschung}

Nach der englischsprachigen Erstveröffentlichung kam es im Rahmen der Reader's Digest-Reihe zu Übersetzungen ins Portugiesische und Schwedische (jeweils 1943) sowie ins Französische, Finnische und Dänische (jeweils 1948), die dem Text noch vor der deutschsprachigen Erstpublikation zu einer gewissen Verbreitung verhalfen und daher erwähnenswert sind. Gerade in den 1950er Jahren war die Erzählung auch für den Abdruck in deutschsprachigen Schul-Lesebüchern beliebt (vgl. Klawiter 1991, S. 311f.). Weitere Verbreitung erfuhr der Text danach erst wieder im Anschluss an den Band Brennendes Geheimnis (1987) der Gesammelten Werke in Einzelbänden (GWE).

Das Interesse der Forschung ist marginal geblieben. Dass der Text mit gelegentlichen Autobiografemen versetzt ist - zu nennen sind der Spaniel Kaspar sowie der Umstand, dass der Erzähler als Schriftsteller identifiziert wird -, bemerkt auch Beck, der Zweigs gleichnamigem Spaniel hier seinen „literarischen Platz“ zugewiesen sieht (Beck 1987, S. 373). Zweigs Erzählung vermengt faktuale und fiktionale Elemente, und wenn seine Texte die Schwierigkeit einer Grenzziehung zwischen „essayistischer“ und „dichterischer“ Prosa aufwerfen, die bereits die frühe Forschung bemerkt hat (Gschiel 1953, S. 49), so gilt dies auch für das vorliegende „Erlebnis“. Randolph J. Klawiter verzeichnet den Text zunächst konsequenterweise noch unter „Essays, Lectures, Reviews“ (Klawiter 1991, S. 311f.), nimmt dies später aber zurück (vgl. Klawiter 1999, S. 3). Von einer „nouvelle morale“ mit der umgekehrten Lehre, wonach das Streben nach Geld ein globaler Faktor von Feindschaften sei, spricht der Kommentar der Pléiade-Ausgabe (Lefebvre 2013, S. 1507).

Der künftigen Forschung eröffnen sich nun mindestens drei Perspektiven: Erstens mag sie sich en détail Zweigs Beiträgen für Reader's Digest sowie deren Kontexten widmen; zweitens kann sie von der Erzählung ausgehend nach dem Thema des Geldes und der Ökonomie in Zweigs Euvre fragen, dem bislang kaum Aufmerksamkeit zuteilwurde; und drittens wird sie jedenfalls dazu angehalten sein, anhand der Typoskripte eine verlässliche Textgrundlage herzustellen und einer breiteren Öffentlichkeit zugänglich zu machen.

\section{Stefan Zweig}

Zweig, Stefan (1937): Begegnungen mit Menschen, Büchern, Städten. Wien u.a.: Reichner.

Zweig, Stefan (1939): Anton, Friend of All the World. The Most Unforgettable Character I Ever Met. In: The Reader's Digest (Pleasantville, NY) 35/210/Oktober/1939, S. 69-72.

Zweig, Stefan (1940): A Lesson from Rodin. In: The Reader's Digest (Pleasantville, NY) 37/220/ August/1940, S. 26-28.

Zweig, Stefan (1941a): What Money Means to Me. In: The Reader's Digest (Pleasantville, NY) 39/231/Juli/1941, S. 39-41.

Zweig, Stefan (1941b): Never Hesitate! In: The Reader's Digest (Pleasantville, NY) 39/231/ Juli/1941, S. 41-43.

Zweig, Stefan (1948): Ein Mensch, den man nicht vergisst. Ein Erlebnis. In: Das Beste aus Reader's Digest 1/November/1948, S. 50-54.

Zweig, Stefan (1981): Das Stefan Zweig Buch. Zsgest. v. Knut Beck. Frankfurt a.M.: S. Fischer.

Zweig, Stefan (1987): Ein Mensch, den man nicht vergißt. Ein Erlebnis. In: Ders.: Brennendes Geheimnis. Erzählungen. GWE. Hg. v. Knut Beck. Frankfurt a.M.: S. Fischer, S. 313-319.

Zweig, Stefan (2004): Besuch bei den Milliarden. In: Ders.: Auf Reisen. Feuilletons und Berichte. GWE. Hg. v. Knut Beck. Frankfurt a.M.: S. Fischer, S. 323-337. 


\title{
Weitere Literatur
}

Beck, Knut (1987): Nachbemerkung des Herausgebers. In: Zweig, Stefan: Brennendes Geheimnis. Erzählungen. GWE. Hg. v. Knut Beck. Frankfurt a. M.: S. Fischer, S. 365-374.

Gschiel, Martha (1953): Das dichterische Werk Stefan Zweigs. Diss. Univ. Wien.

Klawiter, Randolph J. (1991): Stefan Zweig. An International Bibliography. Riverside: Ariadne Press.

Klawiter, Randolph J. (1999): Stefan Zweig. An International Bibliography. Addendum I. Riverside: Ariadne Press.

Lefebvre, Jean-Pierre (2013): Notices et notes. In: Zweig, Stefan: Romans, nouvelles et récits. Bd. II. Hg. v. Jean-Pierre Lefebvre. Paris: Gallimard, S. 1487-1541.

Prater, Donald A. (1989): Stefan Zweig. In: Spalek, John M./Strelka, Joseph (Hg.): Deutschsprachige Exilliteratur seit 1933. Bd. 2, Teil 2. New York, Bern: Francke, S. 1057-1098.

\section{Erzählungen aus dem Nachlass}

\subsection{War er es? (1942)}

\author{
Martina Wörgötter
}

1. Entstehung . . . . . . . . . . . . . . . . . . 292

2. Fassungen . . . . . . . . . . . . . . . . . 293

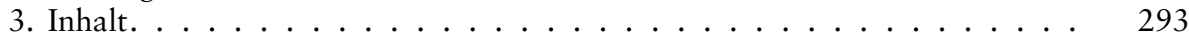

4. Rezeption und Forschung . . . . . . . . . . . . . . . 294

\section{Entstehung}

Die Entstehung von Zweigs Novelle War er es? zu datieren, fällt aufgrund fehlender Dokumentierung schwer; auch der Blick auf die im Literaturarchiv Salzburg vorhandenen Typoskripte (teilweise mit handschriftlichen Korrekturen versehen) lässt keine Rückschlüsse zu. Einen Anhaltspunkt gibt der Schauplatz der Geschichte, Bath, als jener Ort, an den Zweig gemeinsam mit seiner zweiten Frau Lotte im Juli 1939 seinen Wohnsitz verlegt. Jedenfalls handelt es sich bei War er es? - neben der Schachnovelle und Die spät bezablte Schuld (1942) - um eine der letzten Novellen des Autors, die Abrahão Koogan 1942 zusammen in dem Band As três paixões. Três novelas herausbrachte (Guanabara, Rio de Janeiro; unter dem Titel Seria ele?, in einer Übersetzung von Odilon Gallotti und Elias Davidovich). Wie den Briefen zu entnehmen ist, hatte Zweig noch kurz vor seinem Tod den Verleger Koogan bzw. das Haus Guanabara ermächtigt, „meine Autobiographie, meinen Vespucci und meine kleinen Erzählungen zu denselben Konditionen zu publizieren wie das Buch ,Brazil'“" (Zweig an Koogan, 21. Februar 1942, Zweig, Br IV, S. 755). In diesen Tagen geht auch ein Brief an Victor Wittkowski mit der Mitteilung, dass er Koogan gebeten habe, „meine vollendeten und unvollendeten Manuskripte von Ihnen durchsehen zu lassen“ (Brief an Wittkowski, undatiert, verm. 22. Februar 1942, Zweig, Br IV, S. 758).

Eine englische Fassung der Novelle wurde am 3. Juli 1943 unter dem Titel Jupiter in der Zeitschrift Collier's, Springfield, veröffentlicht; deutsch erschien sie zunächst 1973 in einer Rückübersetzung aus dem Englischen, ebenfalls unter dem Titel Jupiter, 